\title{
Hui Yang
}

\author{
A better base editor with fewer off-target changes, from a die-hard Manchester United fan.
}

W hen he was in high school in southern China, a teacher told him, "The 21 st century is the century of life sciences," says Hui Yang, a researcher at the Institute of Neuroscience at Shanghai Institutes for Biological Sciences, which is part of the Chinese Academy of Sciences. It's why he chose to do his $\mathrm{PhD}$ research in biology. Yang studied at Shanghai Jiao Tong University and then completed his $\mathrm{PhD}$ research at the Shanghai Institute of Biochemistry and Cell Biology, focusing on developmental biology. He learned about gene editing while working on a project to generate androgenetic haploid stem cells, but he found the traditional strategies they used to be inefficient.

During a postdoctoral fellowship with Rudolf Jaenisch at the Whitehead Institute, Yang learned about CRISPR-based editing, and his first research project involved stem cells and reprogramming. It was nothing he had previously encountered in his textbooks, he says. "But I quickly fell in love with this." With another postdoc, Haoyi Wang, he worked on generating gene-modified mice and found CRISPR powerful and easier to use than other approaches. In 2014 Yang was recruited back to China as part of the Youth Thousand Talents Program and started a lab. He shifted from creating genetically modified animal models to somatic editing focused on gene editing to one day treat human diseases.

Yang "is a talented young scientist fascinated by new technology," says Mu-ming Poo, who directs the Institute of Neuroscience. "He has the audacity to pursue wild ideas, many of which began with a few sparks in his mind." Yang fearlessly dives into the highly competitive field of gene editing, says Poo, in line with a Chinese saying: "a newborn calf is not afraid of the tiger." Poo met Yang when the latter was a PhD student and they worked on a project involving gene manipulation in monkeys. After Yang returned from his postdoctoral fellowship, he joined the institute, where he enjoys Poo's mentorship. "My hobby is just learning new things and seeking new challenges," says Yang.

In his latest work, Yang and his team present base editor variants with improved fidelity and efficiency. He believes they have potential for clinical use, such as in human

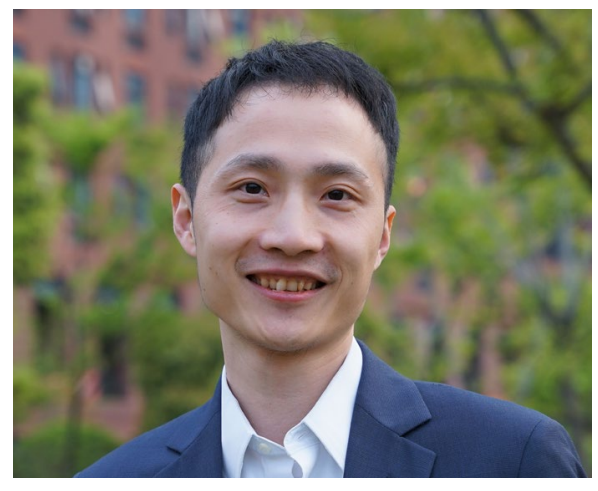

Hui Yang

stem cells and ex vivo applications. But, for these and other clinical applications, "we need more accuracy," he says. CRISPR-Cas-based editing usually involves double-stranded breaks. In base editing, which generally uses the rat cytidine deaminase APOBEC1 fused to the Cas 9 nickase, only single stranded breaks are made. The result at the targeted site is conversion of cytosine to thymine. Yang and his colleagues have tuned base editors: they engineered the deaminase to achieve better expression and nuclear localization, says Yang. Their three base-editing variants generate fewer off-target changes and unwanted insertions and deletions than other base editors.

And they have a narrower base-editing window. "We do not want to correct one mutation and induce another mutation," he says. But when cytosines are near the targeted base, bystander mutations can lead to unwanted on-target effects.

Using structure as a guide, the team focused on engineering the DNA-binding domain of the deaminase. The deaminase has its own DNA-binding domain, but it's not needed; the Cas9 DNA-binding domain can be used instead, says Yang. They looked into which amino acids matter most, chose those, and screened for variants that affect DNA binding but that do not negatively affect the other desired traits: on-target efficiency and low off-target rates. Because the DNA-binding domain clusters with the RNA-binding domain, he believes the variants can be used for DNA and RNA editing. "Some amino acids are key for both," he says. Some variants had higher off-target rates for RNA editing and others for DNA editing, but the researchers managed to find some with good efficiency and low off-target levels for both RNA and DNA editing.

For in vivo editing, he prefers RNA editing. The appeal is the smaller size of these editors and their higher efficiency. To him, RNA editing appears safer than DNA editing, although some concerns remain about non-specific cutting. "But I believe this could also be resolved," he says.

For drug screening in which a broad editing window is acceptable, CRISPR is likely the right choice, says Yang. Base editing's clinical promise is connected to the fact that many diseases are caused by point mutations, he says. He and a former student have founded a company called Hui-Gene Therapeutics to explore gene editing and human disease. Much work remains to be done, especially related to safety, he says.

\section{"My hobby is just learning new things and seeking new challenges."}

Yang often gets ideas for the lab through interaction on social media, usually WeChat, but he prefers face-to-face discussion, he says. In the Jaenisch lab, he liked how valued independent thinking and interaction were and has styled his lab in that vein. Lab members can knock on his door anytime, he says. He helps students and postdocs and encourages more experienced lab members to guide others in designing and doing experiments. In lab meetings, "we just share ideas." One of his students is in a joint program with a Danish university, and he wants to attract others from outside China to the lab. Once a week Yang plays basketball with friends, but he adores soccer. Since high school he's been a die-hard Manchester United fan.

\section{Vivien Marx}

Published online: 19 May 2020

https://doi.org/10.1038/s41592-020-0857-1

Reference

Zuo, E. et al. A rationally engineered cytosine base editor retains high on-target activity while reducing both DNA and RNA off-target effects. Nat. Methods https://doi.org/10.1038/s41592-020-0832-x (2020). 